\title{
PRODUCTION OF PULLULAN BY A THERMOTOLERANT AUREOBASIDIUM PULLULANS STRAIN IN NON- STIRRED FED BATCH FERMENTATION PROCESS
}

\author{
Ranjan Singh ${ }^{1}$, Rajeeva Gaur ${ }^{2 *}$, Soni Tiwari ${ }^{2}$, Manogya Kumar Gaur ${ }^{3}$ \\ ${ }^{1}$ Amity Institute of Microbiol Biotechnology (AIMB), Amity University, Noida (U.P.), Índia; ${ }^{2}$ Department of Microbiology (DST- \\ FIST Supported, Center of Excellence), R.M.L.A. University, Faizabad (U.P.), Índia; ${ }^{3}$ D.G.M. Environment, Balrampur Distillery, \\ Babhanan, Basti (U.P.), India.
}

Submitted: November 02, 2009; Returned to authors for corrections: November 18, 2011; Approved: June 07, 2012.

\begin{abstract}
Total 95 isolates of Aureobasidium pullulans were isolated from different flowers and leaves samples, out of which 11 thermotolerant strains produced pullulan. One thermotolerant non-melanin pullulan producing strain, designated as RG-5, produced highest pullulan $(37.1 \pm 1.0 \mathrm{~g} / \mathrm{l})$ at $42^{\circ} \mathrm{C}$, $\mathrm{pH} 5.5$ in $48 \mathrm{~h}$ of incubation with $3 \%$ sucrose and $0.5 \%$ ammonium sulphate in a non-stirred fed batch fermentor of 6 liters capacity. The two liters of initial volume of fermentation medium was further fed with the 2 liters in two successive batches at $5 \mathrm{~h}$ interval into the fermentor. The sterile air was supplied only for $10 \mathrm{~h}$ at the rate of $0.5 \mathrm{vvm}$.
\end{abstract}

Key words: Aureobasidium pullulans, Pullulan, Non-stirred fed-batch fermentation.

\section{INTRODUCTION}

Pullulan is the generic name given to water-soluble homopolysaccharide that is produced extracellularly by a polymorphic fungus, Aureobasidium pullulans (De Bary) Arnaud. It is a linear $\alpha$-D-glucan connected with $\alpha-1,4$ glycosidic bond mainly of maltotriose repeating units interconnected by $\alpha-1,6$ linkages. The regular alternation of $\alpha$ 1,4 and $\alpha-1,6$ bond results in two distinctive properties, the structural flexibility and enhanced solubility (10). This polysaccharide is of great economic importance with increased application in food, pharmaceutical, agricultural and chemical industries $(3,15)$. Pullulan produces a high viscosity solution at a relatively low concentration and can be used for oxygen- impermeable films and fibers, thickening or extending agent or adhesives or encapsulating agents (11).

Till date, most of the studies have been reported for mesophilic strains of this fungus which produces pullulan up-to level of 30-36 g/1 in flask shake experiments or in fermentor experiments at a temperature range of $24-32^{\circ} \mathrm{C}$ at different physico-chemical conditions. $(3,4,7,8,11,14,17)$. However, till to date no work has been reported for higher production of pullulan above $35^{\circ} \mathrm{C}$.

Non-stirred fermentation system is beneficial over stirred fermentation system because it cuts down the cost and energy. Therefore, strains capable of producing high amount of pullulan in non-stirred fermentation system may be beneficial for commercial production of pullulan. A thermotolerant strain

*Corresponding Author. Mailing address: Department of Microbiology (DST-FIST Supported, Center of Excellence) Dr. Ram Manohar Lohia Avadh University Faizabad. 224001 (Uttar Pradesh). India.; Tel.: +91-9956754873 Fax: +91-5278-246330.; E-mail: rajeevagaur@rediffmail.com 
is better than mesophilic strain at commercial level, because it can sustain high temperature and also cut down the price involved in cooling devices during fermentation. Furthermore, melanin is produced by most of the $A$. pullulans during fermentation. This will make it more difficult to purify polysaccharide after fermentation. Therefore, non-melanin producing strain is another important choice during screening and selection of strain for pullulan production.

In view of the importance of this polysaccharide, an attempt was made to isolate a thermotolerant, non-melanin producing strain of $A$. pullulans from different environmental sources viz. flowers, leaves etc. from Dr. Ram Manohar Lohia Avadh University Campus, Faizabad, Uttar Pradesh, India, and optimized at various physico-chemical as well as nutritional parameters for higher pullulan production.

\section{MATERIALS AND METHODS}

\section{Isolation and Maintenance of Micro-Organism}

A. pullulans was isolated from the flowers and leaves samples collected from nearby university campus. Isolation was done by selective enrichment method as followed by Pollock et al. (13). Flowers and leaves samples were soaked in sterile water for 3 days at $35^{\circ} \mathrm{C}$, and then $0.1 \mathrm{ml}$ was transferred to $10 \mathrm{ml}$ of basal fungal medium ( $\mathrm{pH} 5.0$ ). After 2 days, the turbid cultures were allowed to sit undisturbed for 20 minutes to settle the filaments and aggregates into to the bottom. About $10 \mu \mathrm{l}$ of upper partially clarified culture was spread onto base agar medium plates containing Glucose, 2.0\%; Ammonium Sulphate, 0.06\%; di-Potassium Hydrogen Orthophosphate, 0.5\%; Sodium Chloride, 0.1\%; Magnesium Sulphate, 0.04\% and Yeast Extract, 0.04\% with pH -5.0. (Qualigens Chemicals, Mumbai, Maharashtra, India). Isolates were maintained on the same medium at $4^{\circ} \mathrm{C}$ in slants and sub-cultured monthly. The isolates were identified on the basis of morphological and cultural characteristics using standard identification manuals of fungi $(6,8)$ on its respective medium.

\section{Inoculum Preparation}

Cell suspension was prepared by inoculating $1 \mathrm{ml}$ of $48 \mathrm{~h}$ grown culture in $200 \mathrm{ml}$ nutrient in broth of the same medium and incubated at $42^{\circ} \mathrm{C}$ for $24 \mathrm{~h}$ to achieve active exponential phase of the culture.

\section{Optimization of Fermentation Conditions}

The various process parameters influencing pullulan production by fermentation were optimized individually and independently of the others, therefore, the optimized conditions were subsequently used in all the experiments in sequential order. For optimization, the basal fermentation medium contained glucose $2.0 \%$; ammonium sulphate $0.06 \%$; dipotassium hydrogen orthophosphate $0.5 \%$; sodium chloride $0.1 \%$; magnesium sulphate $0.04 \%$ and yeast extract $0.04 \%$ with $\mathrm{pH}-5.0$ was used for inoculation with $A$. pullulans having $50 \times 10^{7} \mathrm{CFU} / \mathrm{ml}$ and then incubated for different periods viz. $12,24,36,48,60,72,84$ and $96 \mathrm{~h}$ at different temperature viz. $25,30,35,40,42,44$ and $46^{\circ} \mathrm{C}$. For pullulan production nonstirred fed-batch fermentation process was followed. For evaluation of $\mathrm{pH}$, the basic medium $\mathrm{pH}$ was adjusted to 3.0, 3.5, 4.0, 4.5, 5.0, 5.5, 6.0 and 6.5 using either $1 \mathrm{~N} \mathrm{HCL}$ or $1 \mathrm{~N}$ $\mathrm{NaOH}$. For the optimal production of pullulan, the strain may require additional carbon and nitrogen sources with varying concentrations in its growth media. Therefore, the growth medium was supplemented with the carbon sources viz. glucose, fructose, sucrose, lactose and xylose (at the level of $2 \%$ ) along with nitrogen sources viz. ammonium sulphate, yeast extract, sodium nitrate, sodium nitrite and histidin (at the level of $0.5 \%$ ) in the fermentor (YORCO Model No. Y555, YORCO INDIA PVT. LTD. Sahibabad India) of 7 liters capacity with a working volume of 6 liters. The fermentation medium was sterilized at $121^{\circ} \mathrm{C}$ for 15 minutes and incubation was done at $42^{\circ} \mathrm{C}$ with all the other conditions at the optimal levels determined previously. The 2 liters of initial volume of the medium was further fed with 2 liters in two successive batches at every 5 hours interval into the fermentor. The sterile 
air was supplied only up-to $10 \mathrm{~h}$ at the rate of $0.5 \mathrm{vvm}$ (at different incubation periods).

\section{Extraction and Estimation of Pullulan}

After fermentation, the culture medium was heated at $100^{\circ} \mathrm{C}$ in water bath for 15 minutes, cooled to room temperature and centrifuged at $12,000 \mathrm{rpm}$ at $4^{\circ} \mathrm{C}$ for 10 minutes to remove cells and other precipitates. Three milliliters of the supernatant were transferred into a test tube, and then $6 \mathrm{ml}$ of cold ethanol (absolute or $95 \%$ ethanol) was added to the test tube and mixed thoroughly and held at $4{ }^{\circ} \mathrm{C}$ for $12 \mathrm{~h}$ to precipitate the extracellular polysaccharide. After removal of the residual ethanol, the precipitate was dissolved in $3 \mathrm{ml}$ of deionized water at $80^{\circ} \mathrm{C}$ and the solution was dialyzed against deionized water for $48 \mathrm{~h}$ to remove small molecules in the solution. The exopolysaccharide was precipitated again by using $6 \mathrm{ml}$ of the cold ethanol and the residual ethanol was removed, the precipitate was dried at $80^{\circ} \mathrm{C}$ to a constant weight (1). Pullulan weight was measured using electronic balance (Sartorius, U.S.A.) and expressed in $\mathrm{g} / \mathrm{l}$.

\section{Hydrolysis of the Purified Extracellular Polysaccharide and} Assay of Reducing Sugar

To assay the component of the extracellular polysaccharide, the purified precipitate was vacum desiccated to no alcohol by using a vacuum pump, then dissolved in $3 \mathrm{ml}$ deionized water at $80^{\circ} \mathrm{C}$ in water bath. The dissolved substrate was hydrolyzed by incubating the mixture of $0.5 \mathrm{ml}$ of the substrate, $0.4 \mathrm{ml}$ of $\mathrm{Na}_{2} \mathrm{HPO}_{4}(0.2 \mathrm{M})$, citric acid buffer $0.1 \mathrm{M}$ (pH 5.0), and $0.1 \mathrm{ml}$ pullulanase (Sigma Chemicals, U.S.A.) for 21 hour at $40^{\circ} \mathrm{C},(16)$. The released reducing sugar was determined by using the modified D.N.S. method (12) for the confirmation of pullulan.

\section{Statistical Analysis}

Karl Pearson method (Variability) was followed for statistical analysis. All the experiments were done in triplicate and mean values were calculated using standard deviation.

\section{RESULTS AND DISCUSSION}

A total of 95 strains of $A$. pullulans were isolated. Out of these, 11 isolates of $A$. pullulans were screened, produced significant amount of pullulan at $37^{\circ} \mathrm{C}$ within 4 days on incubation. Only one non-melanin pullulan producing strain, designated as RG-5, produced highest amount of pullulan at $42^{\circ} \mathrm{C}$ in $48 \mathrm{~h}$ of incubation (data not shown). Thus, this strain was selected for optimization of physico-chemical parameters for higher pullulan yield.

During preliminary screening, stirred and non-stirred fermentation conditions were evaluated for pullulan production at all the range of temperature from $25^{\circ} \mathrm{C}$ to $45^{\circ} \mathrm{C}$ under aeration. Stirred and non-stirred process produced almost same amount of pullulan in $48 \mathrm{~h}$ of incubation at $0.5 \mathrm{vvm}$ of sterile air supplied for $10 \mathrm{~h}$. Thus, in this text only non-stirred results is presented and discussed in the light of different range of temperature, $\mathrm{pH}$, carbon and nitrogen sources at various concentrations for pullulan production in fed batch non-stirred fermentation process. As simple batch fermentation process did not produce significant amount of pullulan as compared to batch process, hence fed batch process was adopted for this study. Non-stirred fermentation process is always beneficial over stirred fermentation process as it saves energy and requires less amount of cost. This strain of $A$. pullulans is capable of producing satisfactorily high amount of pullulan in non-stirred condition, therefore, can be exploited for commercial production of pullulan.

Furthermore, it is an interesting finding that our isolate of A. pullulans strain RG-5 could tolerate $42^{\circ} \mathrm{C}$ mainly for higher yeast count, responsible for pullulan production. A. pullulans is a ubiquitous fungus of polymorphic characteristics and frequently isolated from different ecosystem viz. terrestrial to aquatic. The various morphological forms protect the fungi for long term existence in all sort of environment. Mostly yeastlike phase is found in broth at a temperature range of $25^{\circ} \mathrm{C}$ to $28^{\circ} \mathrm{C}$. This fungus has been reported to produce yeast-like cells 
at $25^{\circ} \mathrm{C}$ to $35^{\circ} \mathrm{C}$, mainly responsible for pullulan production. Further, the thermotolerant nature of $A$. pullulans for pullulan production has not yet been reported so far. India is a temperate as well as subtropical country, and thus A. pullulans diversity in different climatic conditions may be a reason of this finding. Till date no report is available about thermotolerant strain of $A$. pullulans producing pullulan. During the isolation of $A$. pullulans another pullulan producing fungus, Eurotium chevalieri, was also isolated which produced pullulan but not up to the level of $A$. pullulans. (5).

Besides other parameters in fermentation study stirred and non-stirred conditions are also important parameters which affect pullulan production during fermentation by $A$. pullulans $(4,9,14)$.

\section{Time Course of Pullulan Production and Biomass Yield during}

\section{Fermentation}

Pullulan production is directly related to yeast phase of growth. Yeast-like cells are responsible for pullulan production
(2). Incubation period for pullulan production differs from strain to strain, therefore incubation period has also been evaluated for pullulan production and biomass accumulation. Maximum pullulan production $(23.0 \pm 1.5 \mathrm{~g} / \mathrm{l})$ was recorded at $48 \mathrm{~h}$ of incubation (Figure 1). Further, after $48 \mathrm{~h}$ of incubation, the production of pullulan became stable. This was mainly because the fungus did not produce pullulan degrading enzyme namely pullulanase. Similar trend in pullulan production was also observed by other workers (4). Similarly, maximum biomass production was also observed at $48 \mathrm{~h}$, since formation of biomass directly depends on pullulan formation which indirectly depends on some of the other factors like sucrose concentration and other physico-chemical parameters. Maximum biomass $(21.0 \pm 0.8 \mathrm{~g} / \mathrm{l})$ was obtained at $48 \mathrm{~h}$ of incubation. Maximum pullulan production was achieved when the cells reached their stationary phase which was at $48 \mathrm{~h}$ and beyond this no further growth in the cells were seen, thus production of pullulan became stable.



Figure 1. Effect of Incubation Time on Pullulan and Biomass Production.

\section{Effect of Temperature on Morphology and Pullulan} Production

Fermentation temperature is one of the most important factors for pullulan production affecting yeasts phase of $A$. pullulans growth because change in the morphology adversely affects pullulan production. In this fungus, yeast form of growth is mainly responsible for pullulan production in submerged system of fermentation. It is clearly indicated in Figure 2 that strain could able to produce high amount of pullulan $(25.0 \pm 0.5 \mathrm{~g} / 1)$ at $42^{\circ} \mathrm{C}$. Hence, we can conclude that the highest yeast like cells could be survived and able to produce pullulan at $42^{\circ} \mathrm{C}$. This is an important finding showing the strain as a thermotolerant, which can withstand high temperature along with maximum production of yeast like cells which are mainly responsible for pullulan 
production. Till now, mostly the production of pullulan was done at a temperature range of $24-32^{\circ} \mathrm{C}$ only $(3,4,7,9,11,14,17)$. This may suggest that the optimal temperature for pullulan production by $A$. pullulans vary from strain to strain.

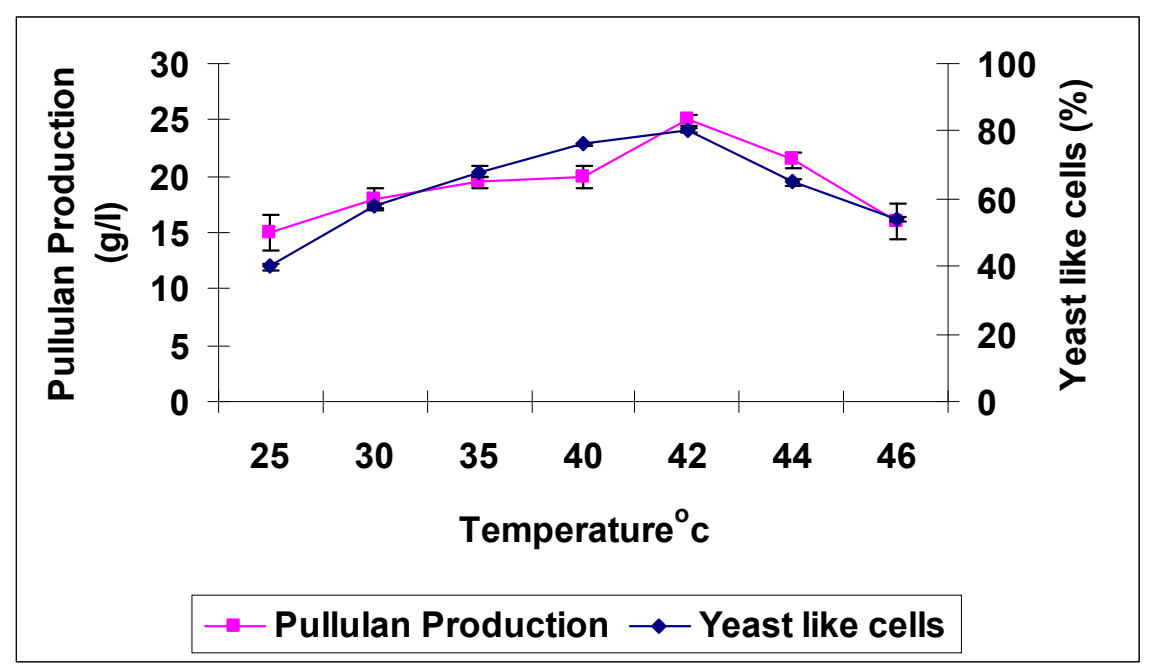

Figure 2. Effect of Temperature on Pullulan Production and Yeast like Cells Formation.

\section{Effect of Initial pH on Pullulan Production}

It has been reported that $\mathrm{pH}$ has profound effect on both the rate of production and the synthesis of pullulan. Different workers have reported pullulan production at different $\mathrm{pH}$ range of $3.0-6.5$ in the medium $(3,4,11)$. The maximum production $(27 \pm 0.5 \mathrm{~g} / \mathrm{l})$ of pullulan in the batch cultivation was recorded at an initial pH 5.5 (Figure 3). This implies that the optimal initial $\mathrm{pH}$ values for pullulan production depend on different yeast strains, composition of the fermentation medium and growth conditions. Therefore, the physiological function of
A. pullulans varies from strain to strain in case of $\mathrm{pH}$ also. This is perhaps due to either special structure of the membrane and cell wall or transport system of the organism along with the change of cytosole $\mathrm{pH}$ due to medium constituents affecting the critical level at specific pka value of medium and ultimately affecting more or less hydrogen ion concentration which in turn affected cell growth or pullulan synthesis. The $\mathrm{pH}$ value reached much lower due to organic acid production by yeast cells after growth of $A$. pullulans, which affects negatively the extracellular polysaccharide production.

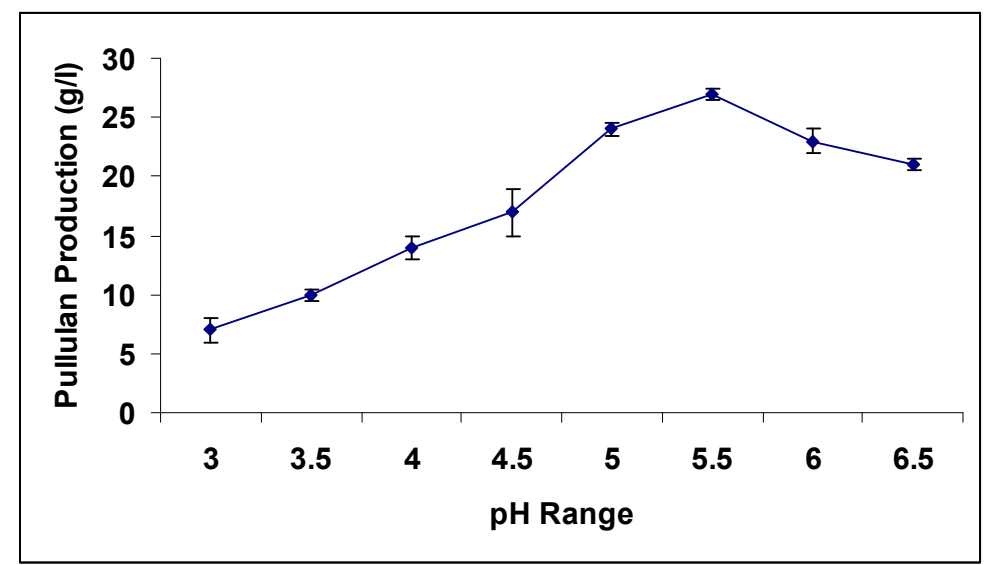

Figure 3. Effect of $\mathrm{pH}$ on Pullulan Production 


\section{Effect of Different Carbon Sources and Concentrations on}

\section{Pullulan Production}

Carbon sources play a vital role in the production of pullulan (4). Sucrose showed best result (33.1 $\pm 1.0 \mathrm{~g} / \mathrm{l})$ among all the carbon sources used at level 2\% (Figure 4). The effect of different concentration of sucrose on pullulan production by this strain was investigated at $42^{\circ} \mathrm{C}$ in the production medium. The optimal concentration of sucrose for pullulan production by this strain was $3 \%(\mathrm{w} / \mathrm{v})$, resulted in pullulan yield of
$35.5 \pm 1.0 \mathrm{~g} / \mathrm{l}$ (Figure 5). Similar carbon source (sucrose used) was also found best for pullulan production as reported by other workers (3). The pullulan production increased with the increase in initial sugar concentration level from 1 to $3 \%(\mathrm{w} / \mathrm{v})$. Further increase of sugar concentration resulted in reduction of pullulan yield. The decline in polysaccharide production encountered with high sugar concentrations in the medium is probably due to osmotic effects, a lower level of water activity as well as plasmolysis events.



Figure 4. Effect of Different Carbon Sources on Pullulan Production

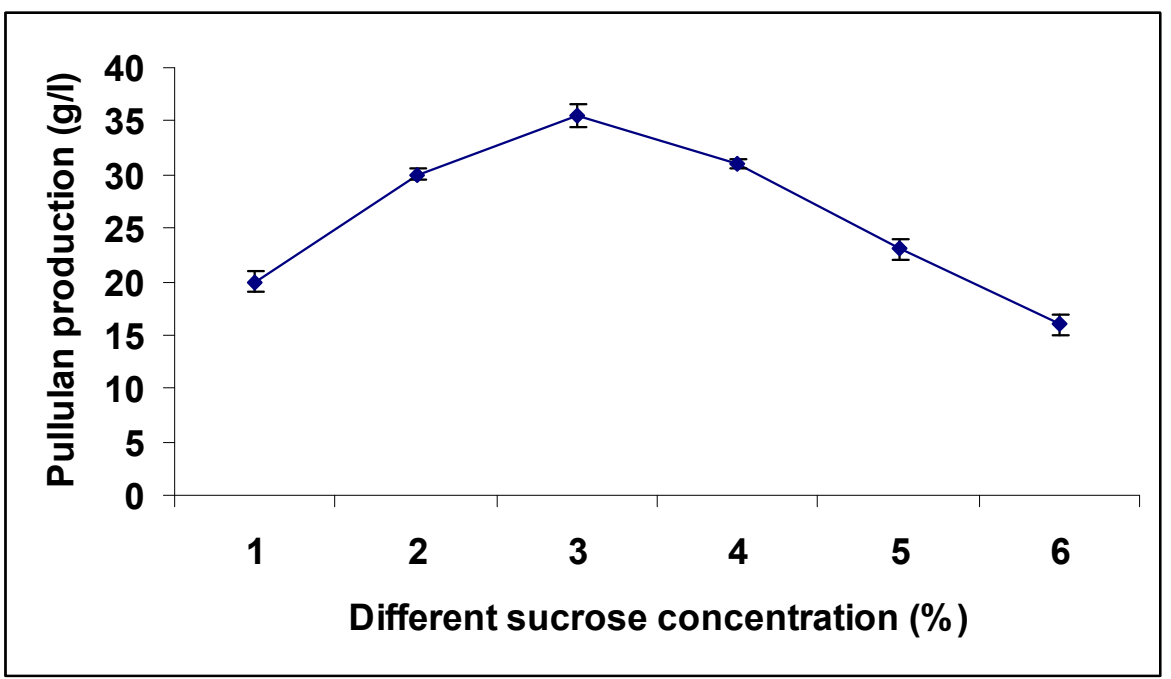

Figure 5. Effect of Different Concentrations of Sucrose on Pullulan Production 


\section{Effect of Different Nitrogen Sources on Pullulan Production}

Different nitrogen sources were also tested to optimize pullulan production in non-stirred fed batch fermentation by this fungus. Among different nitrogen sources (organic and inorganic), the highest pullulan production $(37.6 \pm 1.2 \mathrm{~g} / 1)$ was reported with ammonium sulphate at the level of $0.5 \%$ (Figure 6). In view of this context, different concentrations of ammonium sulphate were further studied. Highest pullulan production $(37.1 \pm 1.0 \mathrm{~g} /)$ was reported at the same concentration of ammonium sulphate. Below and above this concentration $(0.5 \%)$ pullulan production decreased (Figure 7$)$.

Polysaccharide production commended on reaching nitrogen limiting condition, and the yield of pullulan fell when excess ammonium ion were present, even though other conditions were favourable for pullulan production. Various nitrogen sources were optimized for pullulan production by different workers $(3,4)$. Perhaps ammonium ions may be responsible for production of higher biomass resulting in higher pullulan yield.

From the ongoing study it can be concluded that the isolated strain of Aureobasidium pullulans was able to produce higher amount of pullulan by utilizing lesser amount of sugar $(3 \%)$ at $42^{\circ} \mathrm{C}$. This is a novel and an important finding because in industry temperature goes up during fermentation and cooling devices are needed to lower the temperature involving higher cost. Therefore, this strain of A. pullulans can effectively be used for pullulan production in industry to cut down the cost of pullulan. Further, this strain is being improved through mutagenesis, and also through specialized continuous fermentation system using immobilized cells of $A$. pullulans on suitable matrix by manipulating other fermentation conditions. A significant increase of pullulan has also been reported in the above aspects.

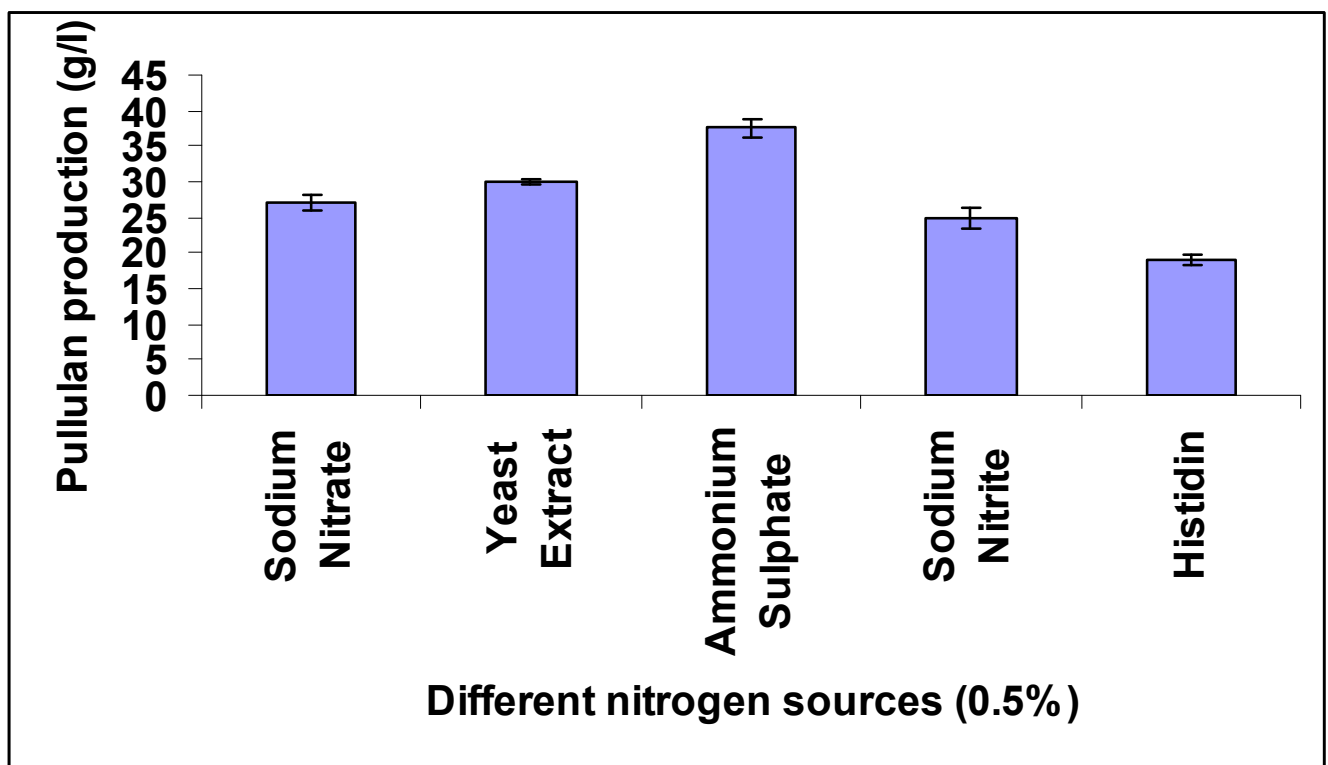

Figure 6. Effect of Different Nitrogen Sources on Pullulan Production 


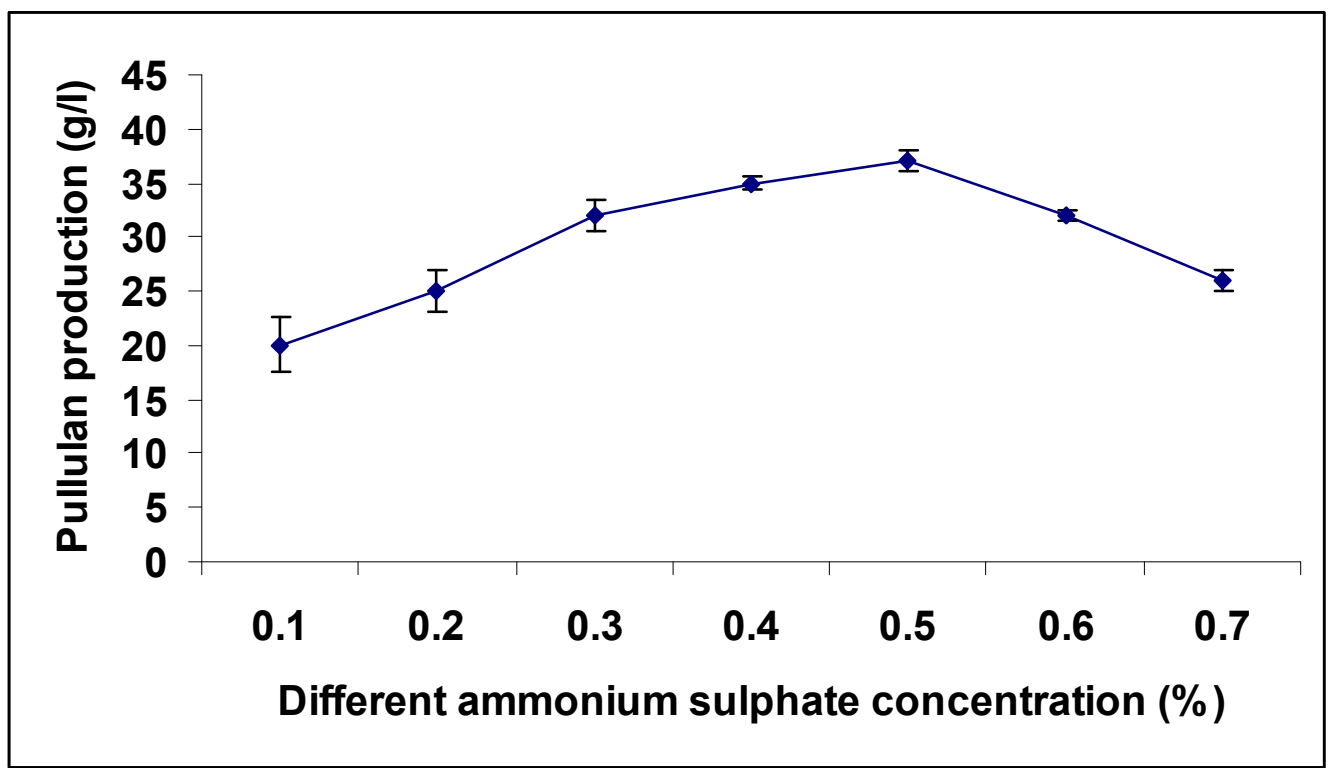

Figure 7. Effect of Different Concentration of Ammonium Sulphate on Pullulan Production

\section{ACKNOWLEDGEMENTS}

Authors are thankful to University Grants Commission, New Delhi, India for the financial support in the form of major research project in the area. Thanks are also extended to the Head, Department of Microbiology, Dr. R. M. L. Avadh University, Faizabad, Uttar Pradesh, India, for providing the research facilities.

\section{REFERENCES}

1. Badr-Eldin, S. M.; El-Tayeb, O. M.; El-Masry, E. G.; Mohamad, O.A.; El-Rahman, O.A.A. (1994). Polysaccharide production by Aureobasidium pullulans: factors affecting polysaccharide formation. World J. Microbiol. Biotechnol. 10, 423-426.

2. Campbell, B. S.; Siddique, A.B.M.; McDougall, B.M.; Seviour, R.J. (2004). Which morphological forms of the fungus Aureobasidium pullulans are responsible for pullulan production? FEMS Microbiol. Lett. 232, 225-228.

3. Cheng, K.C.; Demirci, A.; Catchmark, J. (2009). Effect of Temperature, Carbon Sources, Yeast Extract, and $\mathrm{pH}$ on Pullulan Production by Aureobasidium pullulans. American Soci. Agri. Biol. Eng. 2009 Reno, Nevada, June 21-24.096033.

4. Chi, Z.; Zhao, S. (2003). Optimization of medium and cultivation conditions for pullulan production by a new pullulan- producing yeast strain. Enzyme Micro. Technol. 33, 206-211

5. Gaur, R.; Singh, R.; Tiwari, S.; Yadav, S.K.; Daramwal, N.S. (2010). Optimization of physico-chemical and nutritional parameters for a novel pullulan producing fungus, Eurotium chevalieri. J. Appl. Microbiol. 109, 1035-1043.

6. Gilman, J.C. (1975). A manual of soil fungi. $2^{\text {nd }}$ edition. Oxford and IBS Publishing, New Delhi. India. 50, 109-130.

7. Goksungur, Y.; Ucan, A.; Guvenc, U. (2004). Production of pullulan from beet molasses and synthetic medium by Aureobasidium pullulans. Turk. J. Biol. 28, 23-30.

8. Kurtzmam, C.P.; Fell, J.W. (1998). The yeast: a taxonomic study. The Nertherlands: Elsevier. 45-104.

9. Lazaridou, A.; Biliaderis, C.G.; Roukas, T.; Izydorczyk, M. (2002). Production and characterization of pullulan from beet molasses using a nonpigmented Strain of Aureobasidium pullulans in batch culture. Appl. Biochem. Biotech. 97, 1-22.

10. Leathers, T.D. (2002). Pullulan In: Vandamme, E.J.; De Baets, S.; Steinbuchel, A.; (eds) Biopolymers. Polysaccharides II: polysaccharides from eukaryotes. Wiley-VCH, Weinheim. 6, 1-35.

11. Lee, J.H.; Kim, J. H.; Zhu, I.H.; Zhan, X.B.; Lee, J.W.; Shin, D.H.; Kim, S. K.(2001). Optimization of conditions for the production of pullulan and high molecular weight pullulan by Aureobasidium pulluans. Biotechnol. Lett. 23, 817-820.

12. Lee, J.W.; Yeomans, W.G.; Allen, A.L.; Deng, F.; Gross, R.A.; Kaplan, D.L. (1999). Biosynthesis of novel exopolymers by Aureobasidium pullulans. Appl. Environ. Microbiol. 65, 5265-5271. 
13. Pollock, T.J.; Thorne, L.; Armentrout, R.W. (1992). Isolation of new Aureobasidium strains that produce high-molecular- weight pullulan with reduced pigmentation. Appl. Environ. Microbiol. 58, 877-883.

14. Sena, R.F.; Costelli, M.C.; Gibson, L.H.; Coughlin, R.W. (2006) Enhanced production of pullulan by two strains of Aureobasidium pullulans with different concentration of soyaben oil in sucrose solution in batch fermentation. Brazilian J. Chem. Eng. 23. 507-515.

15. Shingel, K.I. (2004). Current Knowledge on biosynthesis, biological activity, and chemical modification of the exopolysaccharide, pullulan. Carb. Res. 339, 447-460.

16. Su, B. (1986). Techniques for biochemistry and biochemical preparations. Beijing. Science Press. 1, 308.

17. Thirumavalavan, K.; Manikkandan, T.R.; Dhanasekar R. (2008). Batch fermentation kinetics of pullulan from Aureobasidium pullulans using low cost substrates. Bitechnol. 7, 317-322. 